\title{
Análisis del Trabajo Presencial y No Presencial de Profesores y Alumnos bajo el Concepto del Sistema de Transferencia Europeo de Créditos (ECTS)
}

\author{
María Palou y Juan J. Montaño \\ Universidad de las Islas Baleares, Oficina de Convergencia y Armonización Europea, \\ Carretera de Valldemossa, Km. 7,5, 07122 Palma (Islas Baleares)-España \\ (e-mail: maria.palou@uib.es; juanjo.montano@uib.es)
}

\begin{abstract}
Resumen
En este estudio se analizan los hábitos de trabajo del alumnado durante el año académico 20052006 en el primer curso de dos titulaciones, Matemáticas y Psicología, ambas participantes en experiencias de implantación del sistema europeo de créditos (ECTS), realizadas en la Universidad de las Islas Baleares en España. Además, el estudio ofrece información sobre la repercusión de la implementación de las nuevas metodologías de enseñanza-aprendizaje en la carga de trabajo del profesorado. Los resultados indican que hay una sobre-valoración generalizada de las horas de trabajo del alumnado previstas por el profesorado. Los resultados proveen también valiosa información sobre el costo docente que supone la implantación de créditos ECTS y permite mejorar la planificación del volumen de trabajo del alumnado en función del trabajo real realizado.
\end{abstract}

Palabras clave: educación superior, créditos ECTS, trabajo presencial y no presencial

\section{Analysis of the Work Performed by Teachers and Students in Direct Classes and Autonomous Study under the European Credit Transfer System (ECTS)}

\begin{abstract}
This paper analyses the work habits of students during the 2005-2006 academic year in their first year of two careers, mathematics and psychology, both courses included in experiments for implementing the European Credit Transfer System (ECTS) in the University of the Balearic Islands in Spain. The study also provides information on the impact of introducing new teaching/learning methodologies on the workload of the instructors. The results show that the work hours required to the students are overestimated. The results also provide valuable information on the teaching costs derived from the implementation of the ECTS and also allow better planning of the student workloads based on the actual work done by the students.
\end{abstract}

Keywords: higher education area, ECTS system, direct attendance and non attendance 


\section{INTRODUCCIÓN}

La adaptación al Espacio Europeo de Educación Superior (EEES), proceso en el que se encuentran inmersas las universidades de los países de la Unión Europea (UE), tiene como elemento clave la adopción del sistema de créditos European Credit Transfer and Accumulation System (ECTS). La Declaración de Bolonia (Ministros europeos de educación, 1999), que marca el inició de la construcción del EEES, estableció como uno de sus objetivos principales la elaboración de un sistema Europeo de Transferencia de Créditos que asegurase el mayor grado posible de transparencia y calidad en la formación universitaria y que debía estar aplicado antes del año 2010.

El crédito europeo o crédito ECTS nace con los programas europeos de movilidad de estudiantes en el ámbito de la educación superior, fundamentalmente los programas European Community Action Scheme for the Mobility of University Students (Erasmus) y Sócrates, para dar una respuesta a la necesidad de encontrar un sistema de equivalencias y de reconocimiento de los estudios cursados en otros países (Ministerio de Educación, Cultura y Deporte, 2003a). Esta unidad de medida se quiere generalizar para todos los estudiantes de la UE de forma que el trabajo desarrollado por un estudiante sea fácilmente reconocible en cuanto a nivel, calidad y formación en todos los Estados.

El crédito europeo se define en sentido amplio, como la unidad de valoración de actividad académica en la que se integran armónicamente tanto las enseñanzas teóricas y prácticas, así como otras actividades académicamente dirigidas, con inclusión de las horas de estudio y de trabajo que el estudiante debe dedicar para alcanzar los objetivos indicados en el Plan Docente (Ministerio de Educación, Ciencia y Deporte, 2003b). Por tanto, el crédito europeo valora el volumen de trabajo total del estudiante incluyendo el realizado durante los períodos de exámenes u otros posibles métodos de evaluación.

Este nuevo planteamiento introduce sustanciales diferencias con el crédito vigente en España que sobretodo considera el trabajo relacionado con las clases presenciales. A diferencia del actual crédito que mide las horas de clase impartidas por el profesor ( 1 crédito equivale a 10 horas de clase), el crédito ECTS valora el trabajo total de aprendizaje del estudiante. Así, el número de créditos asignados a una materia o asignatura establece la carga real de trabajo que exige al estudiante medio el aprendizaje de esa materia.

Por consiguiente, el proceso europeo de convergencia hacia el EEES requiere la valoración del tiempo y el esfuerzo, que cada asignatura exige al estudiante, en créditos ECTS. Puesto que se establece que un crédito ECTS corresponde a 25-30 horas de trabajo del alumno y que un curso académico equivale a 60 créditos (Paganini, 2002), la dedicación total del alumno no ha de superar las 1800 horas de trabajo anual.

Sin embargo, el cálculo del trabajo o carga académica del estudiante es uno de los aspectos que quedan por resolver. A pesar de haber sido discutido y tratado en el marco del proyecto Tuning, éste no define el procedimiento a seguir para establecer la carga de trabajo del alumno. El mencionado estudio refiere que "el cálculo de dicha carga está en gran medida relacionada con cada disciplina y, por lo tanto, está y tiene que estar determinada por el profesorado". Por otra parte, indica que el concepto de tiempo de aprendizaje, es decir, el correspondiente al número de horas que se supone que un estudiante necesitará como promedio para lograr los resultados del aprendizaje que se especifiquen para un nivel determinado, está influido por al menos los siguientes elementos: la diversidad de tradiciones, el diseño y contexto del currículo, la coherencia del currículo, los métodos de enseñanza y aprendizaje, los métodos de evaluación, la organización de la enseñanza, la habilidad y destrezas del estudiante y el respaldo financiero de dinero público o privado (González y Wagenaar, 2003).

Para adaptar las asignaturas al nuevo modelo, los profesores deben hacer estimaciones para las cuales no cuentan con datos reales que les ayuden a realizar el cálculo del trabajo que debe invertir el alumnado en su asignatura. Este aspecto es crucial, pues sin un sistema de créditos 
fiable y basado en las horas de trabajo del estudiante, que sea considerado de igual modo por todos los interesados, no pueden alcanzarse los objetivos de un espacio europeo de educación superior.

Otro aspecto importante a considerar es la cuantificación del trabajo del profesor tras la implantación del crédito ECTS y los cambios metodológicos que conlleva. Actualmente, la valoración de la carga docente se realiza en términos de horas de clase, de manera que no se tienen en cuenta el número de alumnos matriculados, las horas necesarias para la preparación del material docente, el seguimiento, la evaluación continua, etc. Se desconoce la repercusión que supone la implantación de créditos ECTS en la carga de trabajo del profesorado. Por ello, si consideramos necesario cuantificar las horas no presenciales o de trabajo autónomo del alumnado, no es menos relevante cuantificar la dedicación del profesorado.

En el marco del EEES, las universidades españolas han realizado diversos estudios específicamente dirigidos a la valoración del tiempo y el esfuerzo que exigen al alumnado las asignaturas de distintas áreas de conocimiento. Se pueden mencionar los trabajos realizados en la Escuela Universitaria Politécnica de la Universidad del País Vasco (Guisasola et al., 2006a, 2006b), la Universidad de La Laguna (Herrera et al., 2006) o la Universidad de Valencia (Jano y Ortiz, 2007).

De la misma manera, surgen las investigaciones que tienen por objeto conocer las implicaciones que el sistema ECTS tiene en la planificación y gestión del tiempo de aprendizaje y enseñanza (Yánez, 2006 y Tortella-Feliu et al., 2007). Esta proliferación de estudios pone de manifiesto el interés que despierta entre el profesorado universitario el reto de programar las asignaturas en créditos ECTS.

El estudio que se presenta se ha realizado en el ámbito de las experiencias de implantación del crédito ECTS, contexto considerado óptimo para obtener información sobre la carga real de trabajo promedio de los estudiantes en las diferentes metodologías de enseñanza-aprendizaje (presencial y autónoma) y su repercusión en el costo docente. Concretamente, nos centraremos en los resultados derivados de las experiencias piloto ECTS llevadas a cabo, durante el año académico 2005-2006, en el primer curso de las titulaciones de Psicología y de Matemáticas de la Universidad de las Islas Baleares (UIB).

El objetivo fundamental de este trabajo es realizar una valoración cuantitativa de la carga de trabajo presencial y no presencial que implica la implementación del modelo de Bolonia tanto para el alumnado como para el profesorado. Así mismo, se pretende estudiar las posibles discrepancias entre la estimación realizada por el profesorado y el trabajo real llevado a cabo por el alumnado. Se espera que los resultados obtenidos puedan servir de referencia a las asignaturas que en el futuro tendrán que adaptar su carga de trabajo al EEES.

\section{METODOLOGÍA}

Para alcanzar los objetivos planteados en el estudio, se ha utilizado un muestro no probabilístico de tipo incidental. Se ha trabajado con dos muestras de estudiantes pertenecientes a la UIB. La primera muestra estaba compuesta por 104 estudiantes matriculados en el primer curso de la licenciatura de Psicología, mientras que la segunda muestra estaba compuesta por 24 estudiantes matriculados en el primer curso de la licenciatura de Matemáticas. Finalmente, se han obtenidos los datos referidos al trabajo del profesorado responsable de las seis asignaturas obligatorias (establecidas como tales por la UIB) y troncales (asignaturas establecidas por el Ministerio de Educación y que figuran en todos los planes de estudios independientemente de la Universidad que los imparta) de que consta el primer curso de la licenciatura de Psicología.

En la recolección de la información se ha utilizado la metodología de encuestas. El registro de la dedicación de cada estudiante se ha realizado mediante la aplicación de un cuestionario ad hoc. Para ello, se ha recogido la dedicación semanal (en horas) de cada alumno encuestado durante todo el año académico para cada titulación y asignatura. 
El cuestionario diseñado en el presente estudio recoge información sobre dos aspectos fundamentales:

1. Tiempo de dedicación a la asistencia a actividades de trabajo presencial: las horas de clases teóricas y prácticas, las tutorías (obligatorias o voluntarias) y las actividades de evaluación.

2. Tiempo de dedicación al trabajo autónomo: horas de estudio, preparación de: prácticas, trabajos, resúmenes de lecturas, seminarios, exámenes, obtención de datos, hacer problemas y ejercicios, etc.

Para la recogida de datos relativa a la dedicación del profesorado, se utilizó una hoja de cálculo donde cada profesor registraba semanalmente el tiempo invertido en actividades de tipo presencial (asistencia a clase) y en actividades de trabajo no presencial (preparación de la actividad docente).

El análisis de los datos se centró en el estudio descriptivo del trabajo registrado por el alumnado y por el profesorado. Por una parte, este estudio permite analizar las posibles discrepancias entre el trabajo real del alumnado y el trabajo planificado previamente por el profesorado y, de esta forma, conocer la bondad o precisión de dicha planificación. Por otra parte, nos permite obtener una valoración del volumen de trabajo del profesorado que supone la implantación del crédito ECTS. El tratamiento y el análisis de los datos se realizó mediante la utilización del programa Statistical Package for the Social Sciences (SPSS Inc., 2006).

\section{RESULTADOS Y DISCUSIÓN}

Las tablas siguientes muestran los principales resultados referidos al trabajo registrado y planificado de los estudiantes de las titulaciones de Psicología y Matemáticas, respectivamente. Para cada asignatura y tipo de trabajo (presencial, no presencial y total) se facilita el tamaño de la muestra (N), la estimación del trabajo realizada por el profesorado en horas (Estim.) y en créditos europeos (ECTS), la media aritmética del número de horas de trabajo registrado por el estudiante (m), su desviación estándar (sd) y la precisión de la estimación en términos de porcentaje (Precisión). En este estudio se determinó el valor de un crédito ECTS en 25 horas de trabajo global (presencial y autónomo) del estudiante.

Tabla 1: Trabajo presencial y autónomo del alumnado planificado y registrado en la titulación de Psicología

\begin{tabular}{|c|c|c|c|c|c|c|c|c|c|}
\hline \multirow{3}{*}{ Asignatura } & \multirow{3}{*}{$\mathrm{N}$} & \multicolumn{4}{|c|}{ Trabajo Presencial } & \multicolumn{4}{|c|}{ Trabajo No Presencial } \\
\hline & & \multirow[t]{2}{*}{$\begin{array}{l}\text { Estim. } \\
\text { (ECTS) }\end{array}$} & \multicolumn{2}{|c|}{ Realidad } & \multirow[t]{2}{*}{\begin{tabular}{|l} 
Preci- \\
sión
\end{tabular}} & \multirow[t]{2}{*}{$\begin{array}{l}\text { Estim. } \\
\text { (ECTS) }\end{array}$} & \multicolumn{2}{|c|}{ Realidad } & \multirow[t]{2}{*}{$\begin{array}{l}\text { Preci- } \\
\text { sión }\end{array}$} \\
\hline & & & $\begin{array}{c}\mathrm{m} \\
\text { (ECTS) }\end{array}$ & sd & & & $\begin{array}{c}\mathrm{m} \\
\text { (ECTS) }\end{array}$ & sd & \\
\hline $\begin{array}{l}\text { Análisis de Datos } \\
\text { Anual: } 9 \text { créditos }\end{array}$ & 104 & $\begin{array}{c}65 \\
(2,60)\end{array}$ & $\begin{array}{l}34,55 \\
(1,38)\end{array}$ & 6,51 & 53,15 & $\begin{array}{c}160 \\
(6,40)\end{array}$ & $\begin{array}{l}90,57 \\
(3,62)\end{array}$ & 43,71 & 56,60 \\
\hline $\begin{array}{l}\text { Fundamentos de } \\
\text { Neurociencias } \\
\text { Anual: } 9 \text { créditos }\end{array}$ & 76 & $\begin{array}{c}65 \\
(2,60)\end{array}$ & $\begin{array}{l}43,51 \\
(1,74)\end{array}$ & 6,87 & 66,94 & $\begin{array}{c}160 \\
(6,40)\end{array}$ & $\begin{array}{l}82,78 \\
(3,31)\end{array}$ & 40,82 & 51,74 \\
\hline $\begin{array}{l}\text { Procesos Psicológicos } \\
\text { Básicos } \\
\text { Anual: } 9 \text { créditos }\end{array}$ & 66 & $\begin{array}{c}65 \\
(2,60)\end{array}$ & $\begin{array}{l}53,24 \\
(2,13)\end{array}$ & 5,25 & 81,90 & $\begin{array}{c}160 \\
(6,40)\end{array}$ & $\begin{array}{l}57,00 \\
(2,28)\end{array}$ & 24,94 & 35,62 \\
\hline $\begin{array}{l}\text { Psicología de la } \\
\text { Personalidad } \\
\text { Anual: } 8 \text { créditos }\end{array}$ & 55 & $\begin{array}{c}67 \\
(2,68)\end{array}$ & $\begin{array}{l}61,90 \\
(2,48)\end{array}$ & 9,74 & 92,39 & $\begin{array}{c}133 \\
(5,32)\end{array}$ & $\begin{array}{c}119,20 \\
(4,77)\end{array}$ & 53,55 & 89,62 \\
\hline $\begin{array}{l}\text { Metodología } \\
\text { Experimental } \\
\text { Cuatr: } 6 \text { créditos }\end{array}$ & 105 & $\begin{array}{c}37,5 \\
(1,53)\end{array}$ & $\begin{array}{l}19,83 \\
(0,80)\end{array}$ & 2,49 & 52,88 & $\begin{array}{c}112,50 \\
(4,47)\end{array}$ & $\begin{array}{l}50,05 \\
(2,00)\end{array}$ & 19,96 & 46,59 \\
\hline $\begin{array}{l}\text { Aprendizaje y } \\
\text { Condicionamiento } \\
\text { Cuatr: } 6 \text { créditos }\end{array}$ & 80 & $\begin{array}{c}32 \\
(1,28)\end{array}$ & $\begin{array}{l}16,31 \\
(0,65)\end{array}$ & 2,60 & 50,97 & $\begin{array}{c}75 \\
(3,00)\end{array}$ & $\begin{array}{l}35,41 \\
(1,42)\end{array}$ & 23,19 & 47,21 \\
\hline $\begin{array}{l}\text { Software en Psicología } \\
\text { Cuatr: } 6 \text { créditos }\end{array}$ & 35 & $\begin{array}{c}30 \\
(1,20)\end{array}$ & $\begin{array}{l}21,80 \\
(0,87)\end{array}$ & 3,46 & 72,67 & $\begin{array}{c}120 \\
(4,80)\end{array}$ & $\begin{array}{l}27,42 \\
(1,10) \\
\end{array}$ & 12,74 & 22,85 \\
\hline
\end{tabular}


Aunque existen otras investigaciones que muestran índices de dedicación real ligeramente superiores respecto a la dedicación esperada (Cernuda del Río et al., 2005), los resultados del presente estudio coinciden con los obtenidos por otros trabajos de investigación, los cuales obtienen resultados inferiores a la estimación utilizada como referencia (Jano y Ortiz, 2007).

Tabla 2: Trabajo Total (presencial y autónomo) del alumnado planificado y registrado en la titulación de Psicología

\begin{tabular}{|c|c|c|c|c|c|}
\hline \multirow{3}{*}{ Asignatura } & \multirow{3}{*}{$\mathrm{N}$} & \multicolumn{4}{|c|}{ Trabajo Total } \\
\hline & & \multirow[t]{2}{*}{$\begin{array}{l}\text { Estim. } \\
\text { (ECTS) }\end{array}$} & \multicolumn{2}{|c|}{ Realidad } & \multirow[t]{2}{*}{ Precisión } \\
\hline & & & $\begin{array}{c}\mathrm{M} \\
\text { (ECTS) }\end{array}$ & sd & \\
\hline $\begin{array}{l}\text { Análisis de Datos } \\
\text { Anual: } 9 \text { créditos }\end{array}$ & 104 & $\begin{array}{c}225 \\
(9,00)\end{array}$ & $\begin{array}{c}125,12 \\
(5,00)\end{array}$ & 47,05 & 55,60 \\
\hline $\begin{array}{l}\text { Fundamentos de Neurociencias } \\
\text { Anual: } 9 \text { créditos }\end{array}$ & 76 & $\begin{array}{c}225 \\
(9,00)\end{array}$ & $\begin{array}{c}126,29 \\
(5,05)\end{array}$ & 44,47 & 56,12 \\
\hline $\begin{array}{l}\text { Procesos Psicológicos Básicos } \\
\text { Anual: } 9 \text { créditos }\end{array}$ & 66 & $\begin{array}{c}225 \\
(9,00)\end{array}$ & $\begin{array}{c}110,24 \\
(4,41)\end{array}$ & 27,07 & 48,99 \\
\hline $\begin{array}{l}\text { Psicología de la Personalidad } \\
\text { Anual: } 8 \text { créditos }\end{array}$ & 55 & $\begin{array}{c}200 \\
(8,00)\end{array}$ & $\begin{array}{c}181,10 \\
(7,24)\end{array}$ & 58,90 & 90,55 \\
\hline $\begin{array}{l}\text { Metodología Experimental } \\
\text { Cuatr: } 6 \text { créditos }\end{array}$ & 105 & $\begin{array}{c}150 \\
(6,00)\end{array}$ & $\begin{array}{l}69,89 \\
(2,80)\end{array}$ & 21,37 & 46,59 \\
\hline $\begin{array}{l}\text { Aprendizaje y Condicionamiento } \\
\text { Cuatr: } 6 \text { créditos }\end{array}$ & 80 & $\begin{array}{c}107 \\
(4,28)\end{array}$ & $\begin{array}{l}51,71 \\
(2,07)\end{array}$ & 24,64 & 48,32 \\
\hline $\begin{array}{l}\text { Software en Psicología } \\
\text { Cuatr: } 6 \text { créditos }\end{array}$ & 35 & $\begin{array}{l}150 \\
(6,00)\end{array}$ & $\begin{array}{l}49,22 \\
(1,97)\end{array}$ & 14,33 & 32,81 \\
\hline
\end{tabular}

Tabla 3: Trabajo presencial y autónomo del alumnado planificado y registrado en la titulación de Matemáticas

\begin{tabular}{|c|c|c|c|c|c|c|c|c|c|}
\hline \multirow{3}{*}{ Asignatura } & \multirow{3}{*}{$\mathrm{N}$} & \multicolumn{4}{|c|}{ Trabajo Presencial } & \multicolumn{4}{|c|}{ Trabajo No Presencial } \\
\hline & & \multirow[t]{2}{*}{$\begin{array}{l}\text { Estim. } \\
\text { (ECTS) }\end{array}$} & \multicolumn{2}{|c|}{ Realidad } & \multirow[t]{2}{*}{$\begin{array}{l}\text { Preci- } \\
\text { sión }\end{array}$} & \multirow[t]{2}{*}{$\begin{array}{l}\text { Estim. } \\
\text { (ECTS) }\end{array}$} & \multicolumn{2}{|c|}{ Realidad } & \multirow[t]{2}{*}{$\begin{array}{l}\text { Preci- } \\
\text { sión }\end{array}$} \\
\hline & & & $\begin{array}{c}\mathrm{m} \\
\text { (ECTS) }\end{array}$ & sd & & & $\begin{array}{c}\mathrm{m} \\
\text { (ECTS) }\end{array}$ & sd & \\
\hline $\begin{array}{l}\text { Laboratorio de } \\
\text { Matemáticas } \\
\text { Anual } \\
6 \text { créditos }\end{array}$ & 15 & $\begin{array}{c}58 \\
(2,32)\end{array}$ & $\begin{array}{c}53 \\
(2,12)\end{array}$ & 7,00 & 91,38 & $\begin{array}{c}92 \\
(3,68)\end{array}$ & $\begin{array}{c}152 \\
(6,08)\end{array}$ & 126,00 & 165,22 \\
\hline $\begin{array}{l}\text { Informática } \\
\text { Anual } \\
12 \text { créditos }\end{array}$ & 23 & $\begin{array}{c}126 \\
(5,04)\end{array}$ & $\begin{array}{c}101 \\
(4,04)\end{array}$ & 22,00 & 80,16 & $\begin{array}{c}174 \\
(6,96)\end{array}$ & $\begin{array}{c}80 \\
(3,2)\end{array}$ & 69,00 & 45,98 \\
\hline $\begin{array}{l}\text { Análisis Matemático } \\
\text { Anual } \\
15 \text { créditos }\end{array}$ & 22 & $\begin{array}{c}153 \\
(6,12)\end{array}$ & $\begin{array}{c}139 \\
(5,56)\end{array}$ & 17,00 & 90,85 & $\begin{array}{c}222 \\
(8,88)\end{array}$ & $\begin{array}{c}137 \\
(5,48)\end{array}$ & 82,00 & 61,71 \\
\hline $\begin{array}{l}\text { Álgebra Lineal } \\
\text { Cuatrimestral } \\
7,5 \text { créditos }\end{array}$ & 24 & $\begin{array}{c}83 \\
(3,32)\end{array}$ & $\begin{array}{c}69 \\
(2,76)\end{array}$ & 9,84 & 83,13 & $\begin{array}{l}104,5 \\
(4,18)\end{array}$ & $\begin{array}{c}54 \\
(2,16)\end{array}$ & 37,00 & 51,67 \\
\hline $\begin{array}{l}\text { Matemática Discreta } \\
\text { Cuatrimestral } 6 \\
\text { créditos }\end{array}$ & 22 & $\begin{array}{c}62 \\
(2,48)\end{array}$ & $\begin{array}{c}57 \\
(2,28)\end{array}$ & 9,55 & 91,93 & $\begin{array}{c}88 \\
(3,52)\end{array}$ & $\begin{array}{c}55 \\
(2,2)\end{array}$ & 39,30 & 62,50 \\
\hline $\begin{array}{l}\text { Introducción al } \\
\text { Álgebra Abstracta } \\
\text { Cuatrimestral } \\
7,5 \text { créditos }\end{array}$ & 16 & $\begin{array}{c}77 \\
(3,08)\end{array}$ & $\begin{array}{c}71 \\
(2,84)\end{array}$ & 11,17 & 92,21 & $\begin{array}{l}110,5 \\
(4,42)\end{array}$ & $\begin{array}{l}59,75 \\
(2,39)\end{array}$ & 64,01 & 54,07 \\
\hline $\begin{array}{l}\text { Introducción a la } \\
\text { Geometría } \\
\text { Cuatrimestral } 6 \\
\text { créditos }\end{array}$ & 16 & $\begin{array}{c}60 \\
(2,40)\end{array}$ & $\begin{array}{l}59,50 \\
(2,38)\end{array}$ & 3,22 & 99,17 & $\begin{array}{c}90 \\
(3,60)\end{array}$ & $\begin{array}{l}42,5 \\
(1,7)\end{array}$ & 61,31 & 47,22 \\
\hline
\end{tabular}


Tabla 4: Trabajo Total (presencial y autónomo) del alumnado planificado y registrado en la titulación de Matemáticas

\begin{tabular}{|c|c|c|c|c|c|}
\hline \multirow{3}{*}{ Asignatura } & \multirow{3}{*}{$\mathrm{N}$} & \multicolumn{4}{|c|}{ Trabajo Total } \\
\hline & & \multirow[t]{2}{*}{$\begin{array}{l}\text { Estim. } \\
\text { (ECTS) }\end{array}$} & \multicolumn{2}{|c|}{ Realidad } & $\begin{array}{l}\text { Preci } \\
\text {-sión }\end{array}$ \\
\hline & & & $\begin{array}{c}\mathrm{m} \\
\text { (ECTS) }\end{array}$ & sd & \\
\hline $\begin{array}{l}\text { Laboratorio de Matemáticas } \\
\text { Anual } \\
6 \text { créditos }\end{array}$ & 15 & $\begin{array}{c}150 \\
(6,00)\end{array}$ & $\begin{array}{l}205 \\
(8,2)\end{array}$ & 126,73 & 136,67 \\
\hline $\begin{array}{l}\text { Informática } \\
\text { Anual } \\
12 \text { créditos }\end{array}$ & 23 & $\begin{array}{c}300 \\
(12,00)\end{array}$ & $\begin{array}{c}181 \\
(3,24)\end{array}$ & 76,80 & 60,33 \\
\hline $\begin{array}{l}\text { Análisis Matemático I } \\
\text { Anual } \\
15 \text { créditos }\end{array}$ & 22 & $\begin{array}{c}375 \\
(15,00)\end{array}$ & $\begin{array}{c}276 \\
(11,04)\end{array}$ & 85,82 & 73,6 \\
\hline $\begin{array}{l}\text { Álgebra Lineal } \\
\text { Cuatrimestral } \\
7,5 \text { créditos }\end{array}$ & 24 & $\begin{array}{l}187,5 \\
(7,50)\end{array}$ & $\begin{array}{c}122,26 \\
(4,89)\end{array}$ & 39,95 & 65,20 \\
\hline $\begin{array}{l}\text { Matemática Discreta } \\
\text { Cuatrimestral } 6 \text { créditos }\end{array}$ & 22 & $\begin{array}{c}150 \\
(6,00)\end{array}$ & $\begin{array}{c}111,48 \\
(4,46)\end{array}$ & 43,34 & 74,32 \\
\hline $\begin{array}{l}\text { Introducción al Álgebra Abstracta } \\
\text { Cuatrimestral } \\
7,5 \text { créditos }\end{array}$ & 16 & $\begin{array}{l}187,5 \\
(7,50)\end{array}$ & $\begin{array}{c}130,80 \\
(5,23)\end{array}$ & 67,33 & 69,76 \\
\hline $\begin{array}{l}\text { Introducción a la Geometría } \\
\text { Cuatrimestral } 6 \text { créditos }\end{array}$ & 16 & $\begin{array}{c}150 \\
(6,00)\end{array}$ & $\begin{array}{c}102,00 \\
(4,08)\end{array}$ & 61,67 & 68,00 \\
\hline
\end{tabular}

Entre los resultados más destacados, se puede observar una sobrevaloración generalizada de las horas de trabajo del alumnado previstas por el profesorado que se da tanto en la titulación de Psicología como en la de Matemáticas. De este modo, el promedio de la precisión o bondad de ajuste del trabajo total del estudiante planificado por el profesorado es igual a $54,14 \%$ y $78,26 \%$, en las titulaciones de Psicología y Matemáticas, respectivamente. Esta sobrevaloración ocurre especialmente en el caso de las actividades no presenciales o de trabajo autónomo.

Puesto que un crédito ECTS equivale a 25 horas de trabajo del alumno y que un curso académico consta de 60 ECTS, los alumnos deberían dedicar 1500 horas de estudio para superar cada curso. Sin embargo, según los datos obtenidos, los alumnos de la titulación de Matemáticas confiesan dedicar, por término medio, 371,46 horas menos al año que la dedicación mínima prevista para los ECTS.

Por otra parte, puesto que el curso académico en la Universidad de las Islas Baleares consta de 39 semanas, incluidas las actividades de evaluación, si distribuyéramos las 1500 horas entre el número de semanas del curso tendríamos que cada alumno debería dedicar 38,46 horas a la semana al estudio. En cambio, observamos que el trabajo global realizado por el alumno equivale a 28,93 horas semanales, lo cual está muy por debajo de los límites establecidos en la legislación laboral española (de 35 a 40 horas por semana). Se observa que la mayor discrepancia se halla en la previsión del trabajo no presencial; el esfuerzo realizado en promedio por el alumnado es el $65,86 \%$ del esfuerzo no presencial considerado por los profesores como necesario para superar el curso.

Respecto a los resultados obtenidos en la titulación de Psicología, los alumnos manifiestan trabajar el 55\% de la previsión realizada por el profesorado. Así mismo, se observa que es la estimación del trabajo autónomo la que presenta mayor diferencia con el trabajo real registrado por el alumno. Así los alumnos de Psicología trabajan el 50\% del trabajo autónomo previsto. 
A la luz de estos resultados cabe plantearse si las asignaturas están correctamente dimensionadas y si el trabajo no presencial realizado por el alumno es suficiente. De igual manera, se hace patente la necesidad de un replanteamiento de la exigencia docente.

Por otra parte, se ha registrado el trabajo presencial y no presencial del profesorado. Los resultados obtenidos refuerzan la opinión de que el sistema ECTS tiene implicaciones tanto en la planificación y como en la gestión del tiempo de aprendizaje y enseñanza. Estos resultados coinciden con los obtenidos en estudios anteriores (Salinas, 2007) realizados en la UIB en los cuales, a juicio del profesorado participante, la implantación del crédito ECTS ha supuesto un aumento considerable del volumen de dedicación a las tareas docentes.

La tabla 5 muestra la descripción del volumen de trabajo en horas desarrollado por el profesorado responsable de las asignaturas de la titulación de Psicología. Así, se puede observar que el profesorado ha dedicado un promedio de 119,58 horas a actividades presenciales (tutorías, clases y actividades de evaluación) y un promedio de 170,83 horas a la preparación de la asignatura (trabajo no presencial). Por otra parte, se puede observar que el volumen de la dedicación varía de forma notable en función de las asignaturas. En este sentido, los resultados ponen de manifiesto que la ratio de trabajo del profesorado oscila entre 1,28 y 5,95 horas por cada hora de trabajo del alumnado.

Tabla 5: Trabajo presencial y no presencial del profesorado de la titulación de Psicología

\begin{tabular}{|c|c|c|c|c|c|c|}
\hline & \multicolumn{3}{|c|}{ Trabajo Presencial } & \multirow{2}{*}{$\begin{array}{c}\text { Trabajo } \\
\text { No } \\
\text { Presencial }\end{array}$} & \multirow[t]{2}{*}{$\begin{array}{l}\text { Trabajo } \\
\text { Total }\end{array}$} & \multirow{2}{*}{$\begin{array}{c}\text { Ratio trabajo } \\
\text { profesor / } \\
\text { estudiante }\end{array}$} \\
\hline Asignatura & Clases & Tutorías & Evaluación & & & \\
\hline Análisis de Datos & 32 & 30,5 & 14 & 185 & 261,5 & 2,09 \\
\hline $\begin{array}{l}\text { Fundamentos de } \\
\text { Neurociencias }\end{array}$ & 62 & 16 & 33 & 51 & 162 & 1,28 \\
\hline $\begin{array}{l}\text { Procesos Psicológicos } \\
\text { Básicos }\end{array}$ & 60 & 21 & 10 & 68 & 159 & 1,44 \\
\hline $\begin{array}{l}\text { Psicología de la } \\
\text { Personalidad }\end{array}$ & 104 & 59 & 13 & 420 & 596 & 3,29 \\
\hline Metodología Experimental & 111 & 81 & 8 & 73 & 273 & 5,28 \\
\hline $\begin{array}{l}\text { Aprendizaje y } \\
\text { Condicionamiento }\end{array}$ & 46 & 3 & 14 & 228 & 291 & 5,91 \\
\hline
\end{tabular}

Es importante destacar que con la introducción del sistema ECTS se deberá considerar una nueva fórmula para calcular la dedicación del profesorado. La labor docente también se ve afectada por la introducción del crédito ECTS (Yániz, 2006) de manera que se deberán tener en cuenta sólo las horas de docencia presenciales y las tutorías (Ministerio de Educación y Ciencia, 1985), como sucede actualmente, sino que, al igual que ocurre con el alumnado, también se deberá tener en cuenta el tiempo invertido en la preparación de las asignaturas.

\section{CONCLUSIONES}

Los resultados obtenidos, su análisis y su discusión permiten concluir que:

1- se produce una sobrevaloración constante en las horas de trabajo del alumnado, previstas por el profesorado;

2- esta sobrevaloración ocurre especialmente en el caso de las actividades no presenciales o de trabajo autónomo;

3- se pone de manifiesto que los procedimientos y métodos empleados para la planificación del trabajo del estudiante deben revisarse de cara a disminuir en el futuro la discrepancia observada en este estudio; y

4- la implantación del crédito ECTS pone de manifiesto la necesidad de considerar una nueva fórmula para calcular la dedicación del profesorado que contemple tanto las actividades presenciales como las actividades de preparación de las clases. 
En síntesis, los resultados presentados contribuirán, por una parte, a la revisión de los procedimientos de planificación del trabajo presencial y autónomo del alumnado y, por otra parte, al análisis del costo docente que implicará la implantación generalizada del crédito ECTS en las futuras titulaciones adaptadas al EEES.

\section{AGRADECIMIENTOS}

Este estudio se ha realizado en el marco del Programa de Ayudas del Ministerio de Educación y Ciencia de España (Orden ministerial ECI 9242005, de 21 de Marzo). Se agradece la participación voluntaria del profesorado en las experiencias de implantación de créditos ECTS y el esfuerzo de los todos los estudiantes que han contribuido con el registro del trabajo autónomo y presencial.

\section{REFERENCIAS}

Cernuda del Río, A. y otros cuatro autores. Análisis de los hábitos de trabajo autónomo de los alumnos de cara al sistema de créditos ECTS. http://www.di.uniovi.es/ cernuda/pubs/jenui2005b.pdf. Departamento de Informática, Universidad de Oviedo, 2005. Acceso 8 de Julio (2007).

González, J. y R. Wagenaar (editores); Tuning Educational Structures in Europe. Informe FinalFase uno. Universidad de Deusto/ Universidad de Groningen, (2003) ISBN: 84-7485-893-3. http://www.relint.deusto.es/TUNINGProject/spanish/doc fase1/Tuning\%20Educational.pdf.

Acceso: 1 de Junio (2007).

Guisasola, J., M. Garmendia, J.I. Barragués, y K. Zuza; Cuanto tiempo dedican los estudiantes al estudio de asignaturas básicas de $1^{\circ}$ de ingeniería?. Didáctica de las ciencias experimentales y sociales; ISSN 0214-4379: 20, 89-103. (2006a).

Guisasola, J., M. Garmendia, J.I. Barragués, y K. Zuza. ¿Cuanto tiempo necesita invertir el alumnado para el aprendizaje de una asignatura?: estimación de los créditos ECTS de una asignatura de primer curso de ingeniería. Revista interuniversitaria de formación del profesorado, ISSN 0213-8646: 57, 271-286 (2006b).

Herrera, J. M. y otros seis autores; La experimentación del ECTS en trabajo social: la experiencia desde la Universidad de La Laguna. Acciones e investigaciones sociales, ISSN 1132-192X: 1, 301- 310 (2006).

Jano, D. y S. Ortiz; Estimación del esfuerzo efectivo para superar la asignatura de Estadística Descriptiva en las Licenciaturas de Economía y Administración de Empresa. Relieve: Revista Electrónica de Investigación y Evaluación Educativa, ISSN 1134-4032: 13 (2), 191-202 (2007).

Ministerio de Educación, Cultura y Deporte. La integración del sistema universitario español en el Espacio Europeo de Enseñanza Superior. Documento-Marco. (2003a). Obtenido el 6/6/2007 desde http://www.mec.es/universidades/eees/files/Documento_Marco.pdf

Ministerio de Educación, Cultura y Deporte; Real Decreto 1125/2003, de 5 de septiembre, por el que se establece el sistema europeo de créditos y el sistema de calificaciones en las titulaciones universitarias de carácter oficial y validez en todo el territorio nacional. BOE núm. 224 (2003b)

Ministerio de Educación y Ciencia; Real Decreto 898/1985, de 30 de Abril, sobre Régimen del profesorado universitario. BOE núm. 146 (1985)

Ministros europeos de Educación; Declaración de Bolonia, (1999). Obtenido el 12/6/2007 desde http://www.univ.mecd.es

Paganini, R.; Informe Técnico: El Crédito Europeo y el Sistema Educativo Español. Madrid (2002). Obtenido el 20/6/2007 desde http://www.crue.org/espaeuro/encuentros/credito-vigo2002 .pdf 
Salinas, I. Memoria descriptiva de la experiencia de aplicación de créditos ECTS en el primer curso de Fisioterapia, 2007. Obtenido el 24/6/2007 desde http://www.uib.es/servei/ocihe

SPSS Inc.; SPSS 15.0 para Windows [Programa para ordenador]. SPSS Inc. (Productor). Chicago: SPSS Inc. (Distribuidor) (2006).

Tortella-Feliu, M., M. Servera, y J. Llabrés; Análisis de dos estrategias de enseñanza aprendizaje en la docencia de Terapia de Conducta, Internacional Journal of Clinical and Health Psychology: 7 (2), 547-558 (2007).

Yániz, C.; Planificar la enseñanza universitaria para el desarrollo de competencias. Educatio siglo XXI: Revista de la Facultad de Educación, ISSN 1699-2105: 24, 17-34 (2006). 